\title{
A Sigmoid-Urachal-Cutaneous Fistula after Acute Diverticulitis
}

\author{
Can Balcı', Doğan Gönüllü², Mehmet Lari Gedik², İsmet Hazar1', Elife Kımıloğlu³, Ferda Nihat Köksoy² \\ ${ }^{1}$ Clinic of Urology, Gaziosmanpaşa Taksim Training and Research Hospital, İstanbul, Turkey \\ ${ }^{2}$ Clinic of General Surgery, Gaziosmanpaşa Taksim Training and Research Hospital, İstanbul, Turkey \\ ${ }^{3}$ Clinic of Pathology, Gaziosmanpaşa Taksim Training and Research Hospital İstanbul, Turkey
}

\begin{abstract}
The urachus is an embryonic remnant of communication between the bladder and the umbilicus; it usually obliterates and becomes a fibrous cord, but the incomplete obliteration or partial recanalization leads to the development of anomalies such as urachal cyst or patent urachus. As a consequence of the diverticulitis episode, the sigmoid colon or peridiverticular abscess may adhere to the patent urachus and develop a colourachal fistula with feculent umbilical drainage. Here, we evaluated a case of sigmoid-urachal-cutaneous fistula in the light of the current literature. (JAREM 2015; 5: 83-4)
\end{abstract}

Keywords: Acute diverticulitis, urachus, sigmoid-urachal-cutaneous fistula

\section{INTRODUCTION}

The urachus is an embryonic remnant of communication between the bladder and the umbilicus (1,2); usually, it obliterates and becomes a fibrous cord $(3,4)$, but the incomplete obliteration or partial recanalization leads to the development of anomalies such as urachal cyst or sinus, patent urachus, and urachal diverticula (4). The urachal rest may become infected or ruptured and fistulize to the adjacent organs (2). As a consequence of the diverticulitis episode, the sigmoid colon or peridiverticular abscess may adhere to the patent urachus and develop a colourachal fistula with feculent umbilical drainage, pneumaturia, and fecaluria; the colourachal fistula is very rarely reported in the present literature (1-6).

\section{CASE PRESENTATION}

A 54-year-old male presented with feculent and gassy discharge from his umbilicus, pneumaturia, and blurines of urine; the discharge had started 3 months ago, and his history revealed an undefined abdominal pain at the right quadrant. Physical examination showed only limited periumbilical erythema and feculent discharge. The urinalysis revealed a small amount of leucocytes and erythrocytes, and the microscopic examination showed bacteriuria. The ultrasonography and magnetic resonance imaging showed a urachal remnant containing a cystic lesion and an adjacent mass from the colonic segment. Cystoscopy demonstrated an ostium with feculent drainage, and colonoscopy showed diverticular disease and obstruction at the level of sigmoid colon.

After an infraumbilical midline incision, the exploration of the abdomen revealed a fistulous tract from the umbilicus to the bladder dome in close vicinity with the sigmoid colon. We performed an "en bloc" resection of the involved sigmoid colon, excision of the urachal remnant with the umbilicus and the bladder cuff with end-to-end colonic anastomosis. The bladder was closed in two layers. Pathological examination demonstrated an inflamed fistula tract between the umbilicus, bladder, and sigmoid colon (Figure 1). Written informed consent was obtained from the patient described in this case report.

\section{DISCUSSION}

The urachus is an embryonic remnant of the communication between the bladder and the umbilicus; the persistence of this communication is a 1 in 5000 incidence of urachal anomalies (1). Usually, the urachus obliterates prenatally or, sometimes, during the first year after birth; the obliterated urachal tube forms the median umbilical ligament; in the inner contain a epithelium similar to the urothelium.

Usually, urachal anomalies remain asymptomatic, and most symptomatic anomalies are present in the early childhood period. In a patent urachus, urachal cyst, or urachal sinus, the patient can present with an umbilical discharge or a sign of omphalitis; in cases of infected cysts, they present with an infraumbilical midline painful mass or peritonitis during the rupture of the cyst $(1,4,5)$.

Adenocarcinomas are frequent in urachal rests, and the excision of the urachal rest must be accompanied by bladder cuff excision (5).

\section{CONCLUSION}

Adults with urachal anomalies present with symptoms of complicated urachal cyst; these cysts can fistulize to the adjacent bowel, and the majority of reported entero-urachal fistulae were associated with Crohn's disease. Only few cases of urachal-sigmoid fistulae have been reported (1). Most of these cases were without any obvious colonic pathology; our case is of one of the rarely 


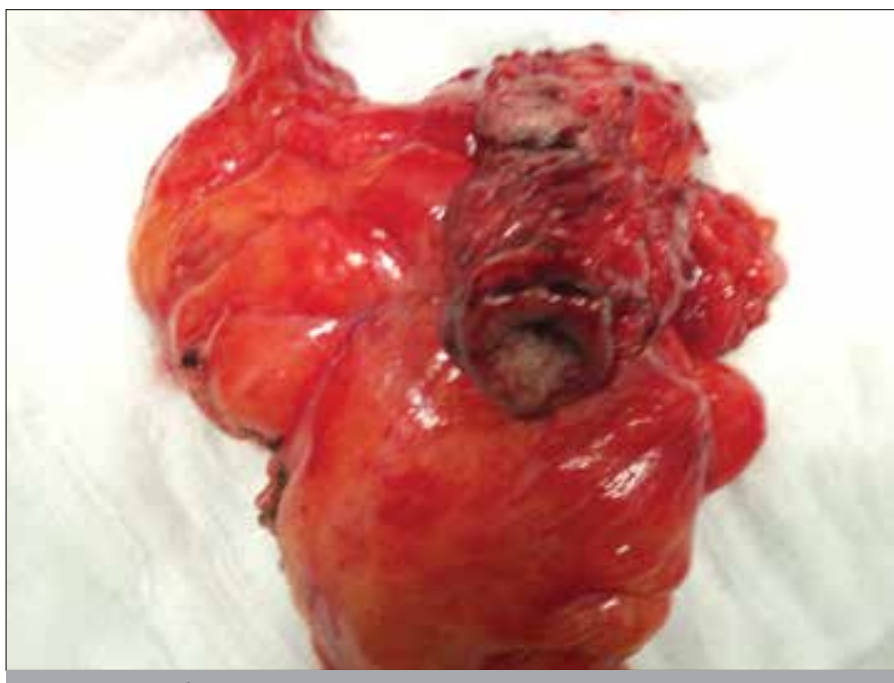

Figure 1. The fistula tract between the umbilicus, bladder, and sigmoid colon

reported sigmoid urachal cutaneous fistula associated with diverticulosis. We suppose that the cutaneous fistula between the sigmoid colon and patent urachus developed after a complicated diverticulitis episode.

Informed consent: Written informed consent was obtained from patient who participated in this case.
Peer-review: Externally peer-reviewed.

Author Contributions: Concept - D.G., C.B.; Design - D.G., C.B., F.N.K.; Supervision - M.L.G., D.G.; Resource - M.L.G., I.H; Materials - E.K.; Data Collection and/or Processing - E.K., D.G., C.B.; Analysis and/or Interpretation - F.N.K., D.G.; Literature Review - E.K., C.B., M.L.G.; Writer - D.G., C.B.; Critical Review - F.N.K., C.B., i.H.

Acknowledgements: We would like to thank to Burak Gönüllü for his active contribution in the translation of this article.

Conflict of Interest: No conflict of interest was declared by the authors.

Financial Disclosure: The authors declared that this study has received no financial support.

\section{REFERENCES}

1. Rapoport D, Ross A, Goshko V, McAuley I. Urachal-sigmoid fistula associated with diverticular disease. Can Urol Assoc J 2007; 1: $52-4$.

2. Peters AL, Kruijer MJ, Wiese H, Verbeek PC. Case report: a colo-urachal-cutaneous fistula in an 88-year-old male. Int J Surg Case Rep 2012; 3: 55-8. [CrossRef]

3. Mutuş MH, Demircan M, Çetin S, Karaman K. Patent Urachus with Stone: A Case Report. JIUTF 2000; 7: 266-8.

4. Yiee JH, Garcia N, Baker LA, Barber R, Snodgrass WT, Wilcox DT. A diagnostic algorithm for urachal anomalies. J Pediatr Urol 2007; 3: 5004. [CrossRef]

5. Nimmonrat A, Na-ChiangMai W, Muttarak M. Urachal abnormalities: clinical and imaging features. Singapore Med J 2008; 49: 930-5.

6. Quek ML, Shapall Al, Daneshmand S. Colourachal fistula in an adult presenting with feculent umbilical drainage. J Urol 2003; 170: 184. [CrossRef] 